\title{
Spatial and temporal traction response in human airway smooth muscle cells
}

\author{
IVA MARIJA TOLIC-NØRRELYKKE, ${ }^{1,2}$ JAMES P. BUTLER, ${ }^{1}$ \\ JIANXIN CHEN, ${ }^{1}$ AND NING WANG ${ }^{1}$ \\ ${ }^{1}$ Physiology Program, Harvard School of Public Health, Boston, Massachusetts 02115, \\ and ${ }^{2}$ Rugjer Bošković Institute, 10000 Zagreb, Croatia
}

Received 12 April 2002; accepted in final form 19 June 2002

Tolić-Nørrelykke, Iva Marija, James P. Butler, Jianxin Chen, and Ning Wang. Spatial and temporal traction response in human airway smooth muscle cells. Am J Physiol Cell Physiol 283: C1254-C1266, 2002. First published June 26, 2002; 10.1152/ajpcell.00169.2002.-Tractions that cells exert on their substrates are essential in cell spreading, migration, and contraction. These tractions can be determined by plating the cells on a flexible gel and measuring the deformation of the gel by using fluorescent beads embedded just below the surface of the gel. In this article we describe the image correlation method (ICM) optimized for determining the displacement field of the gel under a contracting cell. For the calculation of the traction field from the displacement field we use the recently developed method of Fourier transform traction cytometry (FTTC). The ICM and FTTC methods are applied to human airway smooth muscle cells during stimulation with the contractile agonist histamine or the relaxing agonist isoproterenol. The overall intensity of the cell contraction (the median traction magnitude, the energy transferred from the cell to the gel, and the net contractile moment) increased after activation with histamine, and decreased after treatment with isoproterenol. Cells exhibited regional differences in the time course of traction during the treatment. Both temporal evolution and magnitude of traction increase induced by histamine varied markedly among different cell protrusions, whereas the nuclear region showed the smallest response. These results suggest that intracellular mediators of cell adhesion and contraction respond to contractile stimuli with different rates and intensities in different regions of the cell.

cell contraction; adhesion; mechanical stress; cytoskeleton; traction microscopy

MECHANICAL FORCES EXERTED by adherent cells are important for many vital cellular functions, such as contraction, migration, growth, apoptosis, and gene expression $(4,5,6,27)$. A number of methods have been published to calculate tractions at the interface between the cell and the substrate $(1,3,7)$. One of the methods involves plating the cells on a flexible gel with small fluorescent beads embedded under the surface of the gel. As the cell contracts, the gel becomes deformed, and consequently the beads are displaced. The deformation of the gel can be determined from the displacement of the

Address for reprint requests and other correspondence: I. M. TolićNørrelykke, Niels Bohr Institute, Blegdamsvej 17, DK-2100 København Ø, Denmark (E-mail: tolic@nbi.dk). beads. The traction forces can in turn be calculated from the deformation of the gel $(3,7,23)$.

In our previous work (3) we presented an exact solution to the problem of computing the traction field, given the displacement field and the elastic properties of the gel. The method was termed Fourier transform traction cytometry (FTTC). This approach has been used to calculate traction in adherent human airway smooth muscle (HASM) cells (31). By using this method, it was found that the average traction under the cell increases in response to contractile agonists (e.g., histamine), and decreases in response to relaxing agents (e.g., isoproterenol) (31). However, until now it has remained unknown how different regions of the cell respond dynamically to contractile agonists.

An essential step in obtaining reliable traction values is to accurately estimate the displacement fields from images of the fluorescent microbeads in the gel. In our previous paper (3) we described a simple procedure for estimating the displacement field between two images based on cross-correlation. The goal of this paper is 1) to present a substantially improved method for determining the displacement field, and 2) to use the new method to calculate temporal and spatial distributions of tractions in response to pharmacological stimulation. The method presented here generates displacement fields with high spatial resolution, subpixel displacement values, and high accuracy. By applying this method to smooth muscle cells, we found a markedly different response to histamine in different regions of the cell.

\section{METHODS}

\section{Preparation of Polyacrylamide Gel Substrates}

A technique for preparation of polyacrylamide gel disks (7) was modified and used to make flexible gel disks for HASM cells (31). In brief, a mixture of acrylamide (2\%, Sigma), bis-acrylamide $(0.1$ or $0.25 \%$, Sigma), and yellow-green fluorescent polystyrene microbeads (diameter $0.2 \mu \mathrm{m}, 1: 125$ dilution by volume, fluorescein equivalents per microbead $1.1 \times 10^{5}$; Molecular Probes) was added to glass coverslips.

The costs of publication of this article were defrayed in part by the payment of page charges. The article must therefore be hereby marked "advertisement" in accordance with 18 U.S.C. Section 1734 solely to indicate this fact. 
The droplet of solution was covered by a small circular coverslip (no. 1, 12-mm diameter; Fisher). The whole assembly was turned upside down so that the microbeads would move toward the surface of the gel close to the coverslip by gravity. Only the beads very close to the apical surface of the gel $(<0.5 \mu \mathrm{m})$ were later used for imaging. After polymerization (about $45 \mathrm{~min}$ ), the circular coverslip was removed. Because the cells did not attach to uncoated gel surfaces, type-I collagen $(0.2 \mathrm{mg} / \mathrm{ml})$ was attached to the surface of the gel covalently after activation of the gel with succinimidyl-6(4'-azido-2'-nitrophenylamino)hexanoate (27). The collagen layer was very thin $(<0.1 \mu \mathrm{m})$; thus it did not change the thickness nor the elastic properties of the gel. Gel disks were typically $50-70-\mu \mathrm{m}$ thick and $\sim 1 \mathrm{~cm}$ in diameter.

Polyacrylamide gel exhibited a nearly ideal elastic behavior for large strains (30\%; Ref. 7). The elastic modulus (Young's modulus) of the gel was determined to be $865 \pm 30$ $\mathrm{Pa}$ (mean $\pm \mathrm{SE}$ ) and 1,255 $\pm 80 \mathrm{~Pa}$ for the mixture containing 0.1 and $0.25 \%$ bis-acrylamide, respectively, using the methods described in Wang et al. (31). Poisson's ratio of the gel was taken to be 0.48 . Some investigators reported a value close to 0.5 for the Poisson's ratio of polyacrylamide (7), whereas others used a value of $0.3(18,23)$. Changing the value of Poisson's ratio from 0.48 to 0.3 in the calculations induced only slight changes $(2-3 \%)$ in the traction fields.

\section{Cell Culture and Experimental Protocol}

HASM cells were isolated from tracheal muscle of lung transplant donors, approved by the University of Pennsylvania Committee on Studies Involving Human Beings (26). The cells were serum deprived in IT medium (i.e., the medium containing $5.7 \mu \mathrm{g} / \mathrm{ml}$ insulin and $5 \mu \mathrm{g} / \mathrm{ml}$ transferrin) for 2 days before the experiments. Cells at passage 3-6 were plated on a gel disk in the same serum-free IT medium and allowed to spread for $6 \mathrm{~h}$. A plastic dish with a gel disk in medium was mounted on the microscope stage with temperature control set to $37^{\circ} \mathrm{C}$. The cells were stimulated with graded doses of either histamine $(0.1 \mu \mathrm{M}$ at $t=0,1 \mu \mathrm{M}$ at $t=$ $2 \mathrm{~min}$, and $10 \mu \mathrm{M}$ at $t=4 \mathrm{~min})$ or isoproterenol $(0.01 \mu \mathrm{M}$ at $t=0,0.1 \mu \mathrm{M}$ at $t=3 \mathrm{~min}, 1 \mu \mathrm{M}$ at $t=5.5 \mathrm{~min}$, and $10 \mu \mathrm{M}$ at $t=8.5 \mathrm{~min}$ ). The cells were then left for 10-15 min. Finally, trypsin (2\%) was added to detach the cells from the substrate. Trypsin cleaves proteins on the carboxyl side of lysine or arginine residues. Care was taken not to disturb the dish when trypsin was added. The cell was observed to be gradually rounded up by trypsin and then partially detached from the substrate. Gentle flushes of medium using a catheter connected to a syringe completely detached the cell from the gel surface. The experiment was terminated when the cell in the field of view dissociated from the gel, thereby leaving the gel with no surface tractions.

\section{Microscopy}

Images of the cells were taken both with phase contrast to visualize the cells and with 470-nm illumination to excite the beads, which fluoresce at $515 \mathrm{~nm}$. Phase contrast and fluorescence images were taken interchangeably every $20 \mathrm{~s}$. The size of the images was $1024 \times 1280$ pixels. One pixel was equivalent to an area of $0.17 \times 0.17 \mu \mathrm{m}$. There were typically a few thousand beads per image. The image of a bead occupied 3-4 pixels in diameter. Because the bead size $(200 \mathrm{~nm})$ was below the resolution of light microscopy, the image of a bead was the point spread function of the combined imaging system. Neighboring beads were $\sim 10-30$ pixels apart.

\section{Determination of Displacement Fields}

The process of the determination of the traction field consists of two main parts, the determination of the displacement field from a pair of images and the computation of the traction field from the displacement field. In each of our experiments we recorded a sequence of images. We estimated the displacement field (and later the traction field) between each image and the reference image. The reference image was the image of a force-free gel, i.e., the image taken after the cell was released from its adhesions by trypsin. We used the image correlation method (ICM) for the determination of the displacement field between a pair of images.

Image correlation method. Consider a small window area in the first image (image $A$ ). The aim is to find the matching window in image $B$, i.e., the position to which the window from image $A$ has moved in the time interval between recordings of the two images. The window from image $A$ is compared with windows from the same neighborhood in image $B$. For each of these windows a similarity measure is calculated. The window from image $B$ that has the highest similarity measure with the window from image $A$ is selected as its matching window. A variety of similarity measures can be constructed (see ref. 28). We have chosen to use the crosscorrelation function of two windows.

In calculations of the cross-correlation we use the correlation theorem, which says that the Fourier transform of a correlation of two functions is the product of the Fourier transform of one function and the complex conjugate of the Fourier transform of the other function

$$
\mathrm{FT}_{2}\left[\operatorname{Corr}^{\prime}(G, H)\right]=\mathrm{FT}_{2}(G) \mathrm{FT}_{2}(H)^{*}
$$

where $\mathrm{FT}_{2}$ denotes the two dimensional Fourier transform, Corr' is the correlation of two functions, $G$ and $H$ are two functions (two images in this case), and the asterisk denotes complex conjugation. The mean is removed from each function before calculating the correlation.

We form the normalized two-dimensional cross-correlation function between the two images

$$
\operatorname{Corr}(G, H)=\frac{\operatorname{Corr}^{\prime}(G, H)}{\sqrt{\max \left[\operatorname{Corr}^{\prime}(G, G)\right] \max \left[\operatorname{Corr}^{\prime}(H, H)\right]}}
$$

The cross-correlation function is the similarity measure between the window from image $A$ and windows in image $B$ at various space lags. Coordinates of the peak of the correlation function constitute the displacement vector of the window from image $A$.

For the calculation of the correlation functions we utilized the two-dimensional fast Fourier transform (FFT) algorithm in MATLAB (MathWorks). Even though the images are represented by relatively large $(1,024 \times 1,280)$ matrices, using the correlation theorem and the FFT algorithm makes the computations very fast.

The hierarchical algorithm. At the beginning of the image processing, the pair of images is corrected for relative translational shift. The cross-correlation between the two images is calculated by using Eqs. 1 and 2. The coordinates of the peak of the normalized correlation function are identified, and image $B$ is translated with respect to image $A$ by that uniform displacement.

Next, the displacement field between the two corrected images is calculated starting from a low spatial resolution and refining the calculation to higher and higher resolution. Images are first divided into large distinct windows of equal size. The displacement of each window is calculated as described above for the whole images. Image $B$ is then distorted 
by this displacement field. The procedure is repeated until for all the windows from image $A$ the best match is found in image $B$.

At each succeeding level, the spatial resolution of the displacement vectors is twice that of the previous level. Having an initial approximate displacement field obtained at some level, we distorted image $B$ at the next level by the displacement field from the previous level. Therefore, at each new level, we look for the residual displacements which, when added to the initial ones, give a refined result. The whole procedure is repeated until the desired spatial resolution is obtained (e.g., the centers of windows spaced by $16 \times$ 16 pixels). The hierarchical algorithm is depicted in Fig. 1.

Checkpoint. To reduce errors in the displacement field, a checkpoint is introduced at each level. A pair of windows has to pass all the tests described below; otherwise, the displacement of that window is set to an undefined value. The tests used are the following: 1) Are the windows completely aligned? [i.e., is the peak of the cross-correlation at the position $(0,0)$ ?] ; 2$)$ Is the displacement of the window smaller than half of the window size? At each level we only look for small "corrective" displacements. Large displacements may result in an error from accidentally found high correlation between distant windows after a long unsuccessful search; 3) Does the peak value of the cross-correlation between a pair of windows exceed a certain threshold (usually 0.5)?; 4) Is the maximum intensity in each window higher than a certain threshold? Using this test question we ignore the displacement vectors in windows with no beads; and 5) Is the minimum intensity in each window lower than a certain threshold? This question is used to discard the displacements of windows that are occupied by a large cluster of beads.
Polynomial fitting. After the displacement vectors that did not pass any of the tests are discarded, the rest of the displacement field is fitted to a third-order polynomial. The values of the polynomial in the points for which the displacement value was undefined are taken as the displacements. To obtain subpixel displacement values, the peak of the cross-correlation function at the final level is found by fitting the values of the function in the immediate neighborhood of the pixel with the highest value to a secondorder polynomial.

Filtering. Finally, the displacement field is passed through a low-pass filter such that the high-frequency displacements with the period less than one-tenth of the cell length are filtered out. The assumptions behind designing the filter are 1) the windows used for the calculation of displacements were usually 2.7 or $5.3 \mu \mathrm{m}$ wide; therefore it was not possible to accurately detect displacements that change fast over the window size, i.e., displacements with the period smaller than about $10 \mu \mathrm{m}$; and 2) noise in the displacement field gets amplified when calculating the traction field; a filtering procedure is a simple and well-defined way to smooth the data and reduce noise; the smoothing is done at the level of the displacement field, so that the calculation of the traction field from the final displacement field remains exact.

The result is a uniform discretized displacement field between the two images, with the desired spatial resolution. In most of our calculations the final window size was 32 pixels. The spatial resolution, i.e., the distance between the centers of neighboring windows, was 16 pixels $(2.7 \mu \mathrm{m})$. The optimal spatial resolution of the final displacement field, as well as the thresholds used at the check points, had to be defined by the user. These parameters depend strongly on the proper-

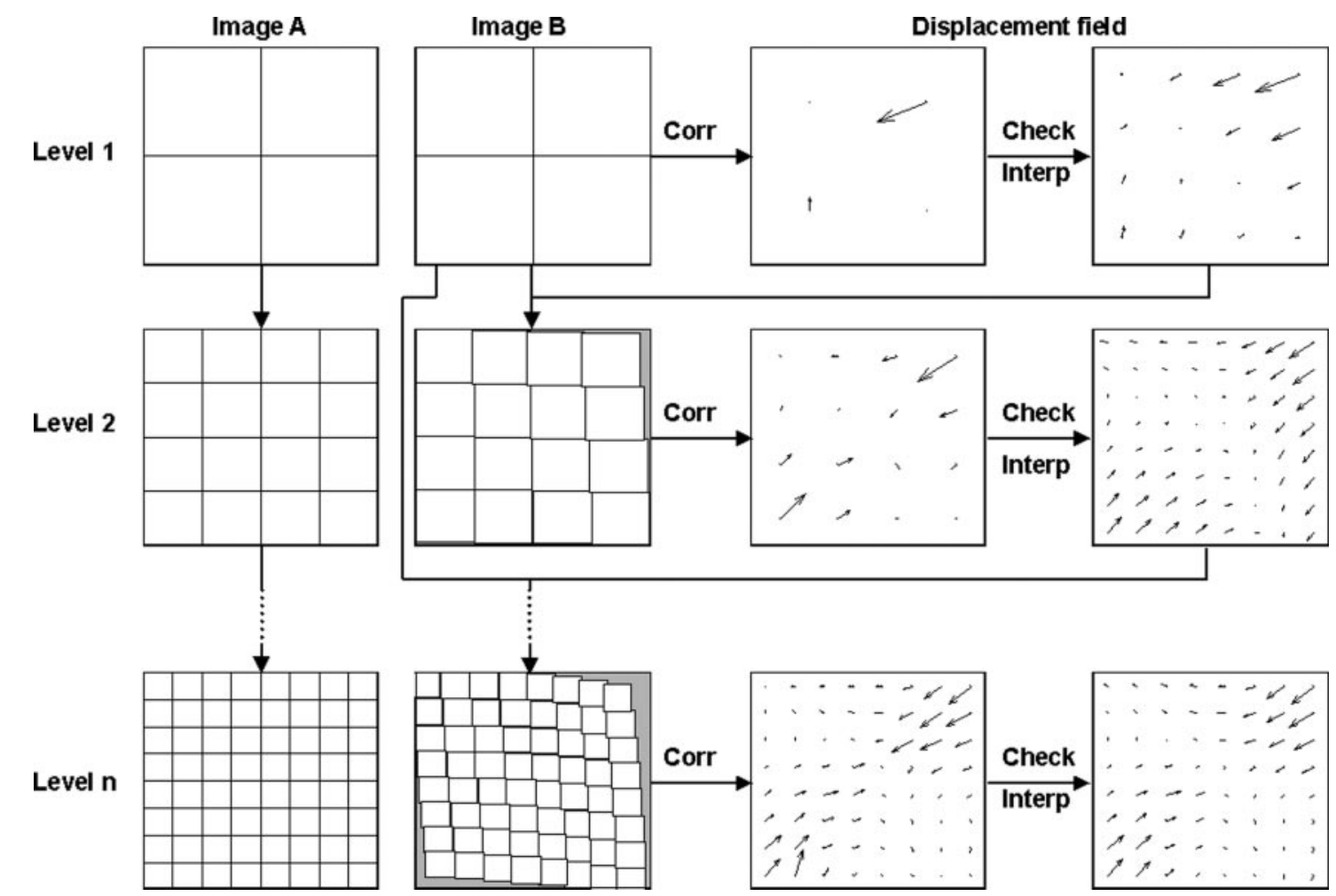

Fig. 1. Flow chart of the image correlation method (ICM). At each level the displacement field is estimated by using image $A$ and image B. Image $B$ is deformed by the displacement field calculated at the previous level. Succeeding levels have finer spatial resolution and smaller window size. Corr, calculation of cross-correlation; check, checkpoint; interp, interpolation. 
ties of the particular images and the degree of the gel deformation.

\section{Performance Tests of the ICM}

To test the accuracy and precision of the ICM, we simulated undeformed and deformed images. First, an undeformed image was constructed, assuming the following: 1) the distribution of the beads in the image is a Poisson distribution; 2) each bead occupies one pixel; 3) the value of each pixel that represents a bead is a random number uniformly distributed on the interval $(150,255)$ (this interval was chosen because in real images pixels occupied by beads had such intensities); and 4) the values of all other pixels are random numbers uniformly distributed on the interval $(0$, 20 ), resembling background noise. Beads in a real image occupied 3 to 4 pixels in diameter. For simplicity, the simulated beads were chosen to be only 1 pixel to avoid introducing a number of new parameters needed to describe a larger image of a bead (e.g., the shape of the intensity distribution at the bead location, the level of noise, etc.).

Second, a deformed image was constructed by displacing each bead from the undeformed image by $u(x)$ pixels in $x$ direction and 0 in $y$ direction. The displacement function $u(x)$ was chosen to be a sawtooth function, beginning at 0 at the left edge of the image, with amplitude 10 pixels, and with variable period. After constructing a pair of images in such a way, the images were used as the input to the ICM with varying window sizes. The displacement fields were calculated and compared with the values of $u(x)$.

\section{Calculation of Traction Forces, Contractile Moments, and Strain Energy}

The traction field was calculated from the displacement field using FTTC, as described in Butler et al. (3). This calculation is based on the Boussinesq solution for the displacement field on a surface of a semi-infinite solid, given the surface tractions (17). Both unconstrained FTTC and constrained FTTC were used. Note that unconstrained FTTC uses the whole displacement field for the calculation of the traction field, whereas in constrained FTTC only the displacements inside the investigator-determined cell boundary are used. The spatial resolution of the traction maps was the same as that of the displacement maps $(2.7 \mu \mathrm{m})$. The temporal resolution was $40 \mathrm{~s}$ (fluorescence images were collected every $40 \mathrm{~s}$ ).
For each traction field contractile moments and strain energy were calculated, following the definitions from Butler et al. (3). The ratio of the contractile moment along the principal axis of traction $\left(\mathrm{M}_{x x}\right)$ to the net contractile moment (M) was used as an index of the polarity of cell contraction. The net contractile moment and the strain energy were used as measures of the overall intensity of cell contraction.

\section{RESULTS}

Figure 2 shows a pair of images of the gel with cultured HASM cells. Images were taken 10 min after the beginning of the experiment in which the cells were stimulated with increasing doses of histamine. Image $A$ is a phase-contrast image of a HASM cell attached to the surface of the polyacrylamide gel coated with collagen I. Image $B$ is a fluorescence image of the same field of view. The distribution of fluorescent microspheres (Fig. 2, white dots) is fairly uniform and dense. The beads can be seen as 3- to 4-pixel-wide bright areas in the enlargement of a small region of the image.

\section{Determination of the Displacement Field}

Figure 3 shows the displacement field between $\mathrm{im}$ age $B$ from Fig. 2 and the reference image (the image of fluorescent beads taken after the cell detached from the gel following treatment with trypsin), obtained by the ICM. The absolute magnitudes of the displacements (in $\mu \mathrm{m}$ ) are represented by colors. The mapping between the displacement values and different colors is shown by the color scale. The color map was chosen in such a way that the isodisplacement curves are easily visualized. Figure 3 also shows the direction of displacements.

The largest displacements were under the cell, and they were especially large (up to $2.5 \mu \mathrm{m}$ ) close to the cell ends. Most of the displacement vectors pointed towards the center of the cell, i.e., towards the position of the nucleus, where they decreased to zero. The displacement fields of the same cell calculated from images taken at different times, as well as the displacement fields of other cells, showed similar features.
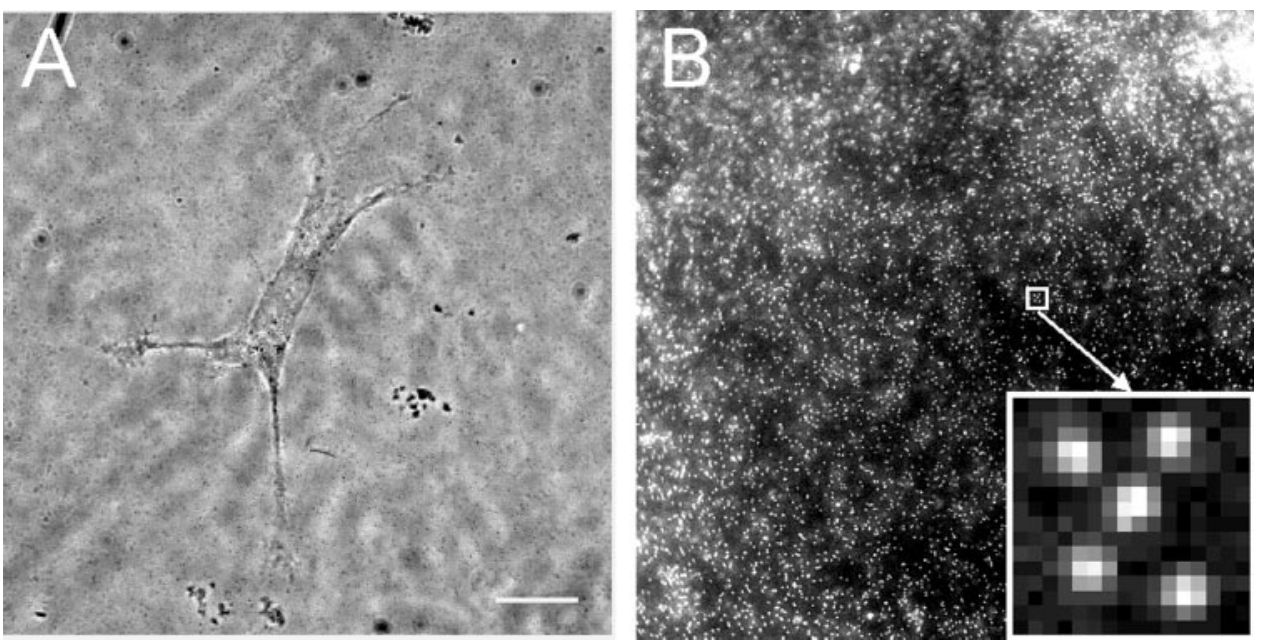

Fig. 2. Images of the gel with cultured human airway smooth muscle (HASM) cells. Images were taken $10 \mathrm{~min}$ after the beginning of the experiment in which the cells were stimulated with increasing doses of histamine. A: phase contrast image of a cell. $B$ : fluorescence image of the microbeads below the gel surface (white dots). Inset shows a magnified $2.7 \times 2.7 \mu \mathrm{m}^{2}$ region from image $B$, which contains 5 fluorescent microbeads. Young's modulus of the gel was $1,300 \mathrm{~Pa}$. Scale bar in $A, 20 \mu \mathrm{m}$. 
Fig. 3. Displacement field calculated from the image in Fig. $2 B$ and the image of the traction-free gel, using ICM. Colors show the magnitude of the displacements in micrometers (see color scale). Arrows show the direction and relative magnitude of the displacements. The displacements were calculated on a grid with a spacing of $2.7 \mu \mathrm{m}$. For visual clarity, the displacement arrows in the figure are spaced by $6 \mu \mathrm{m}$. The value of the color in each pixel was interpolated from the values calculated on the $2.7-\mu \mathrm{m}$ grid. The cell boundary, as drawn by hand from the phase contrast image, is shown by the white line. Large displacements (top right) were probably due to the presence of another cell.

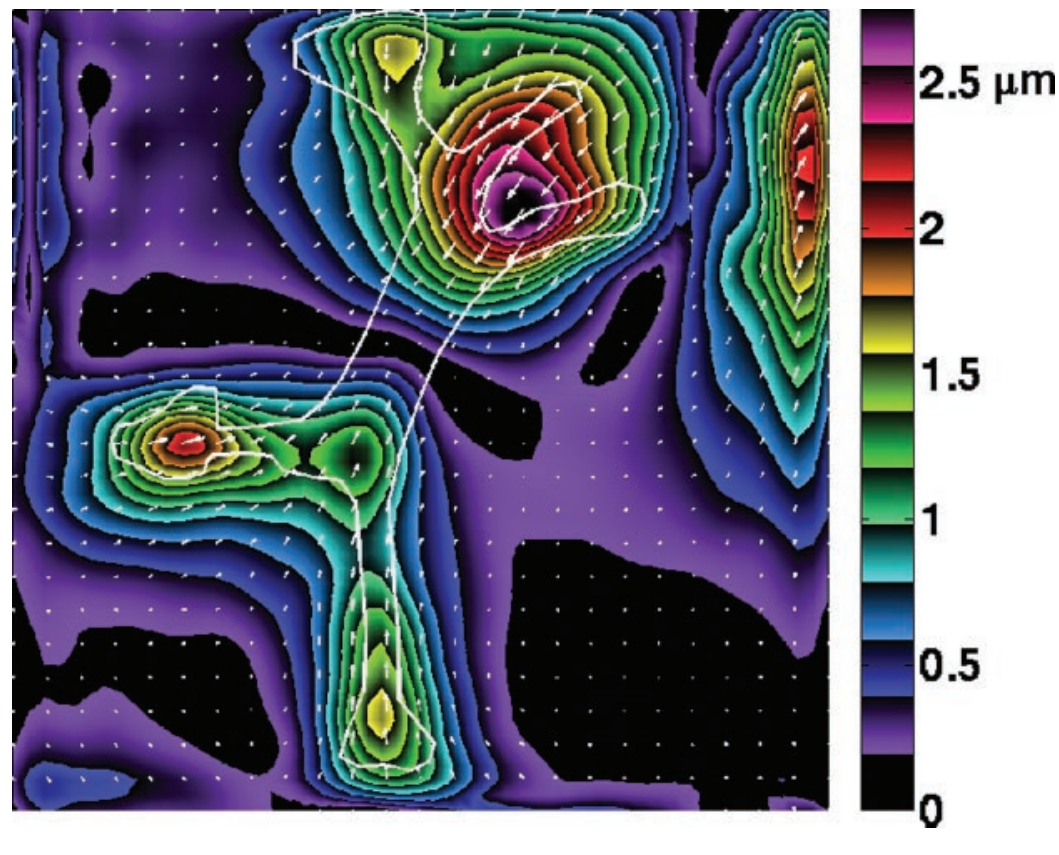

However, in a small number of displacement fields we found displacements pointing away from the cell (see Fig. 3). These were probably because of another cell outside of the field of view, which was also contracting.

\section{Results of Monte Carlo Simulations of Displacement Fields}

The results of the simulations to test the accuracy and precision of the ICM are shown in Fig. 4. Figure $4 A$ shows the mean absolute error of the calculated displacements as a function of the number of beads in the image. The mean absolute error of the calculated displacements is the mean of the absolute difference between the assigned displacement $u(x)$ and the displacement calculated by the ICM. The mean absolute error of displacements was rather large ( 1.5 to 3 pixels) for bead numbers near 100. As the number of beads grew, the error decreased. The error increased again as the number of beads approached the total number of pixels in the image. The error was larger when using windows of $64 \times 64$ pixels than with $32 \times 32$ windows. With a large number of beads in the image (around $10^{5}$ ) and the $32 \times 32$ windows, the error was $0.23 \pm$ 0.03 pixels. These conditions may be considered close to the conditions of our experiments, although the number of the beads in real images was much lower. Recall that in the simulated images, each bead occupied 1 pixel, whereas in real images each bead covered an area of 3 to 4 pixels in diameter. Therefore the number of the beads in the simulation does not correspond directly to the number of the beads in the real image, but the way the error depends on the number of the beads is presumably the same.

Figure $4 B$ shows the mean absolute error of calculated displacements as a function of the relative frequency of the displacement function $u(x)$. The relative frequency was defined as the ratio of the length of the simulated image in $x$ direction and the period of the displacement function $u(x)$. For relative frequency $=1$, the results are equivalent to the results in Fig. $4 A$. As the frequency increased, the error increased much faster than the frequency and became particularly high $(\sim 20$ pixels for relative frequency $=8)$ for $64 \times 64$ windows. This is not a surprise, because the simulated images consisted of $512 \times 512$ pixels, and therefore there could be only 8 distinct $64 \times 64$ windows along the $x$-axis of the image. When the relative frequency of the displacements was 8 , the values of the displacements passed through one whole period within a $64 \times$ 64 window. The displacement vector of a window was defined as the displacement of the window's center point, which was 0 in this case. The fact that the displacements passed through one whole period over a window means that there were many beads displaced by a nonzero value of pixels. Therefore ICM gave as the result the most frequent, usually far from zero displacement. On the other hand, the errors of the displacements were small if the window size was smaller than half of the period of the displacement function $u(x)$. For example, small errors were found in the simulations with the $32 \times 32$ windows and the period of the displacement function larger than 64 pixels, which is equivalent to the relative frequencies smaller than $512 / 64=8$ (see Fig. $4 B$ ).

\section{Traction Fields of HASM Cells}

Figure 5 shows the traction field calculated from the displacement field shown in Fig. 3. In Fig. 5A, unconstrained FTTC was used, whereas in Fig. $5 B$ constrained FTTC was used [this variation constrains the tractions outside of the cell boundary to zero (3)].

The visual representations of the traction maps were generated in a way similar to the displacement field in Fig. 3. Here, the absolute magnitudes of the tractions 

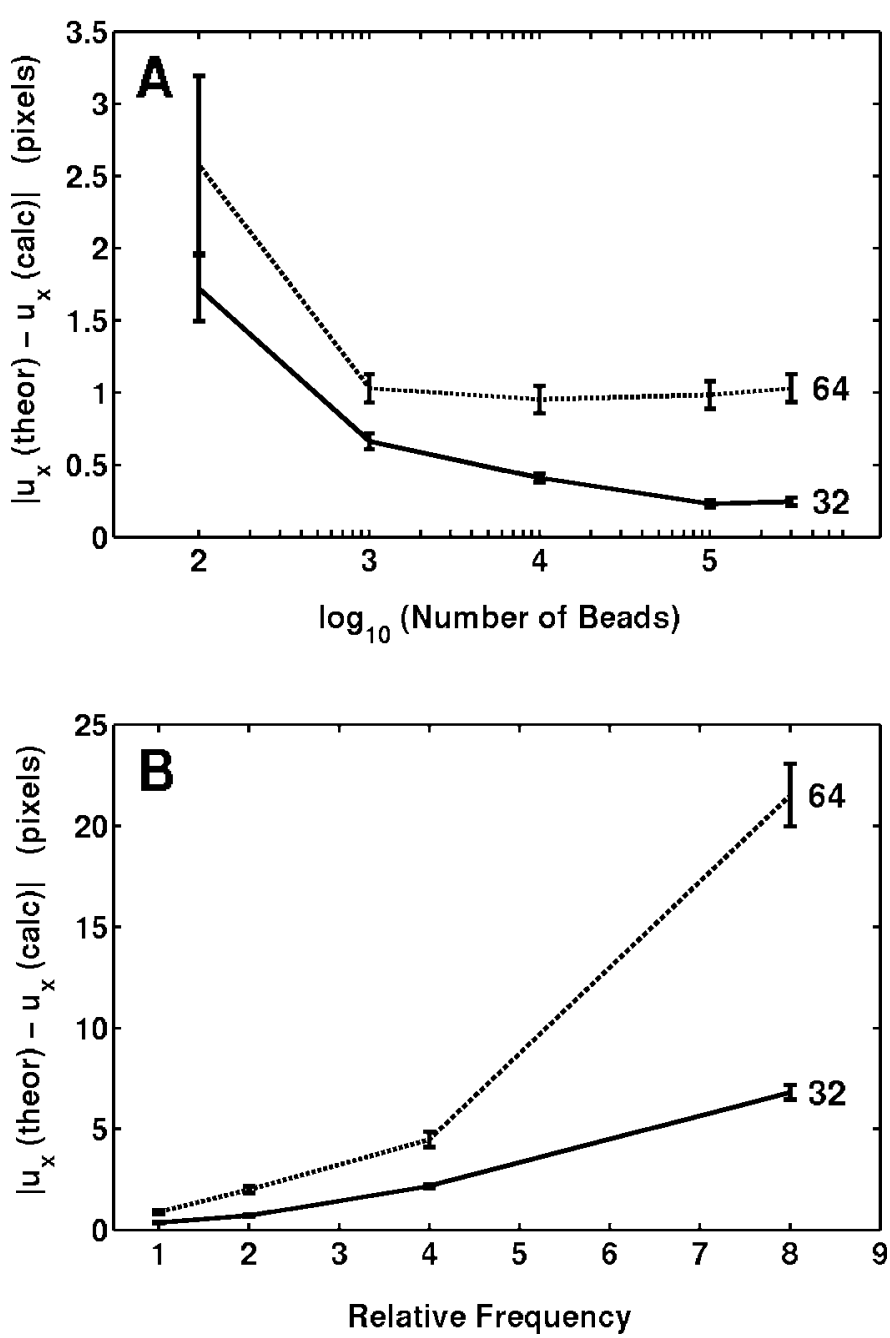

Fig. 4. Results of Monte Carlo simulations of deformed images. The displacement field between a pair of images was calculated by using ICM with $64 \times 64$ pixel windows (dashed lines) and $32 \times 32$ windows (solid lines). A: mean absolute error of calculated displacements as a function of the number of beads in the image. The relative frequency of the displacement function $u(x)$ was $1 . B$ : mean absolute error of calculated displacements as a function of the relative frequency of $u(x)$. The number of the beads was $3.3 \times 10^{4}$. The size of the images was $512 \times 512$ pixels. Error bars represent SE.

(in $\mathrm{Pa}$ ) are represented by colors. The isotraction curves (23) are easily visible. The figure also shows the direction of tractions and the boundary of the cell (Fig. 5A).

In Fig. 5, $A$ and $B$, large tractions were found at the tips of the protrusions, up to about $200 \mathrm{~Pa}$. Those traction vectors pointed towards the nucleus. Tractions were close to zero under the nucleus. The two traction fields from Fig. 5 are similar, yet different in some aspects. We used both of them in the subsequent analysis, treating them as two complementary solutions to the same problem (3).

Figure 6 shows more examples of traction fields generated by HASM cells. Constrained FTTC was used in the calculations. The top row contains two traction fields of the same cell, whereas the bottom row contains traction fields of another cell. Traction fields in
Fig. 6, $A$ and $C$, are baseline traction maps calculated from the images taken at time 0 , i.e., just before adding the first dose of histamine. Fig. $6, B$ and $D$, show the traction fields 10 min later during the same experiment.

\section{Tractions from a Single Traction Field Display a Log-Normal Distribution}

Histograms of the magnitudes of tractions from a single traction field showed skewed distributions with mean values small compared with the range of traction magnitudes (Fig. 7). This kind of distribution was observed at different time points $(0,3,5,7$, and $10 \mathrm{~min})$ in the three cells from Figs. 5 and 6, and in more than 5 other cells (not shown). Here, we present a histogram of traction magnitudes of one cell at time 0 in Fig. $7 A$ and at 10 min in Fig. $7 B$. Insets in Fig. 7, $A$ and $B$, show that the logarithm of traction magnitude was well fit by a normal distribution. Thus the distribution of the traction magnitudes was well fit by a log-normal distribution.

\section{Different Regions of a Cell Respond to Stimulation at Different Times and With Different Intensities}

After analyzing a set of traction vectors from one cell at a given time, we next explored how the traction in a small region of a cell evolved as the cell contracted. We chose 6 small square areas of $16 \times 16$ pixels $(2.7 \times 2.7$ $\mu \mathrm{m}^{2}$ ) under the cell from Fig. $2 A$ : five areas each in a different protrusion, and one under the nucleus. The areas were labeled $N, N E, E, S, W$, and $C$, and their centers are shown in Fig. $8 A$. Figure $8 B$ shows the magnitude of tractions exerted in those six areas as a function of time. Before stimulation with histamine, the traction in all six chosen areas was low $(<30 \mathrm{~Pa})$. The first increase in traction was visible after $2 \mathrm{~min}$.

It is interesting to note the variation in the evolution of traction in the six areas shown. Area $C$ did not develop any significant traction during the experiment. Between 2 and 4 min there was an increase in traction in areas $N, N E$, and $S$, which lie inside of the three protrusions that determine the long axis of the cell. Traction in areas $W$ and $E$ began to increase only after $4 \mathrm{~min}$. After $10 \mathrm{~min}$ the traction in areas $N E, E$, and $S$ saturated at values between 80 and $100 \mathrm{~Pa}$, whereas the traction in areas $W$ and $N$ were $140-180 \mathrm{~Pa}$ and had not yet reached a plateau. Similar results were observed in more than 10 other cells that had more than two protrusions. Because a small area inside of a cell protrusion can be chosen in many ways, we used instead the mean traction in each cell protrusion for statistical analysis, as follows.

Cell protrusions vary widely in size, shape, and position with respect to the cell nucleus; thus the protrusion regions were determined manually. A typical protrusion area was $\sim 100 \mu \mathrm{m}^{2}$. The mean traction was calculated for each protrusion at each time point. We found that the mean traction varied markedly between different protrusions of the same cell. For each cell the 

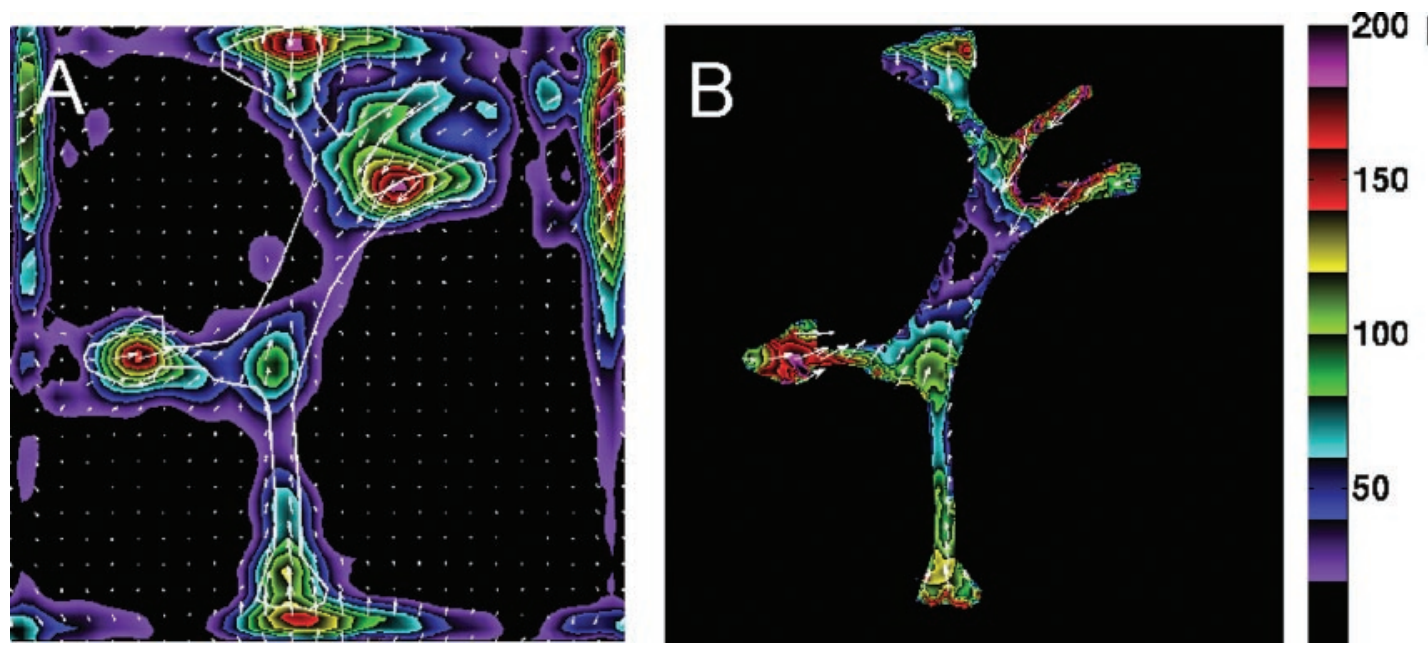

Fig. 5. Traction field calculated from the displacement field shown in Fig. 3, using $(A)$ unconstrained Fourier transform traction cytometry (FTTC) and $(B)$ constrained FTTC. Colors show the magnitude of the tractions in Pa (see color scale). Arrows show the direction and relative magnitude of the tractions. The traction arrows in the figure are spaced by $6 \mu \mathrm{m}$, whereas the tractions were calculated on a grid with a spacing of $2.7 \mu \mathrm{m}$. The cell boundary is shown by the white line in $A$. Note that the cell boundary was not used in unconstrained FTTC, whereas it was used in constrained FTTC where the tractions outside of the cell are constrained to be zero.

protrusion that exerted the largest traction at the end of the experiment was identified, as well as the protrusion with the smallest traction. The difference between the mean traction in the strongest and the weakest protrusion of 10 cells at $t=11$ min was $119 \pm 80 \mathrm{~Pa}$ (mean $\pm \mathrm{SD}$ ); the weakest protrusion exerted $43 \pm 23 \%$ of the traction in the strongest protrusion. Figure $9 \mathrm{~A}$ shows the development of traction in the strongest and in the weakest protrusion of 14 cells (14 strongest protrusions and 14 weakest protrusions were ana- lyzed; however, there is no data for some cells at certain time points due to, e.g., a blurred image or a faster termination of the experiment). The traction in the strongest protrusions was significantly different from the traction in the weakest protrusions ( $t$-test, $P<0.05)$ at all times except for $t=-1 \mathrm{~min}$. Results from the experiments in which the cells were treated with the bronchodilator isoproterenol are shown in Fig. $9 B(n=5$ cells $)$. The traction in the strongest protrusions was significantly different from the traction in
Fig. 6. Traction fields of two HASM cells. Each row shows tractions from one cell. $A$ and $C$ : traction fields at time $O$ (baseline), i.e., just before adding the first dose of histamine. $B$ and $D$ : tractions $10 \mathrm{~min}$ later during the same experiment. Traction fields were calculated using constrained FTTC. Tractions larger than $50 \mathrm{~Pa}$ are shown by the arrows. Young's modulus of the gel was $1,300 \mathrm{~Pa}(A$ and $B)$ and $870 \mathrm{~Pa}(C$ and $D)$. Scale bar, $20 \mu \mathrm{m}$.
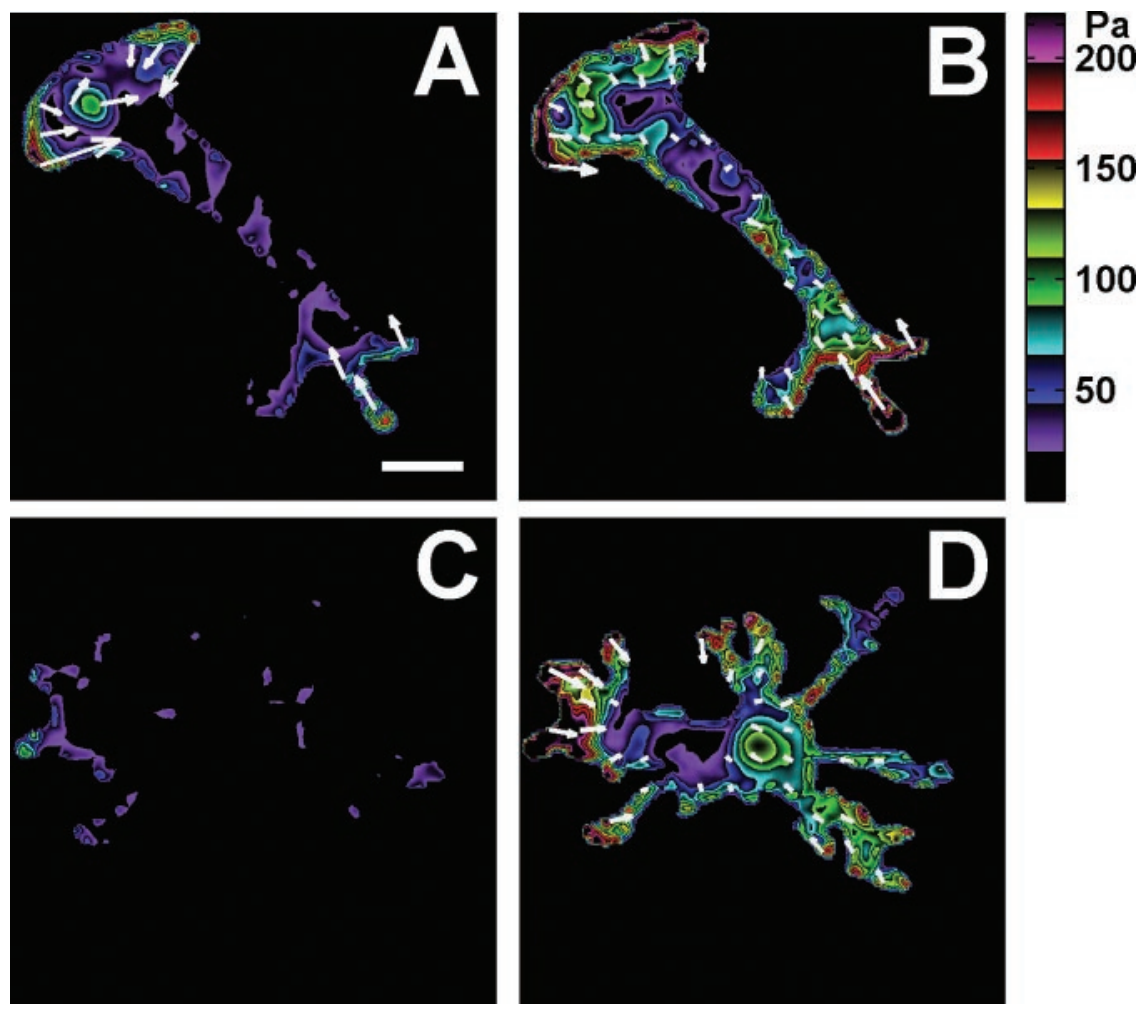

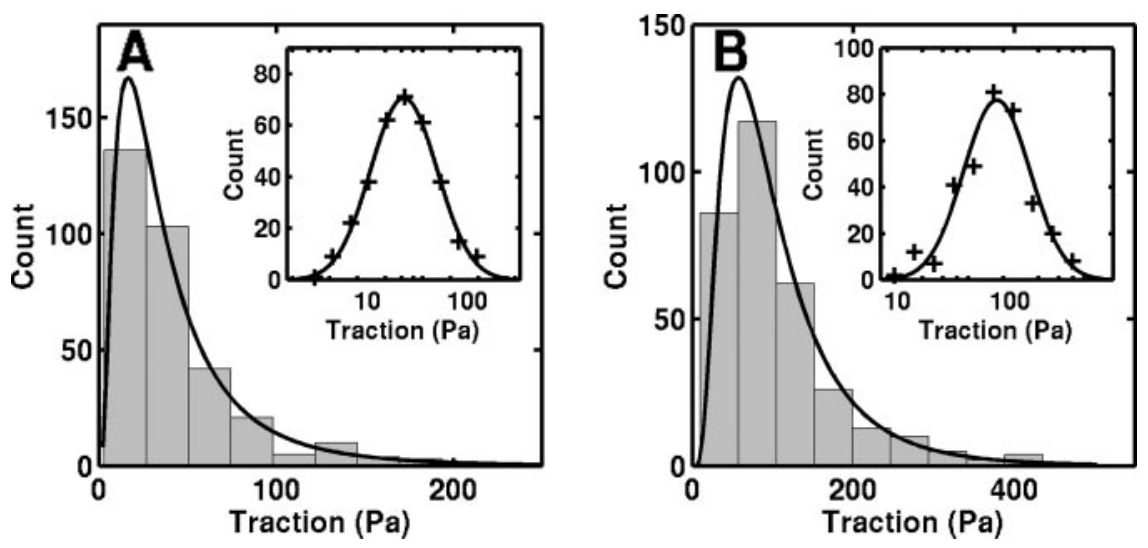

Fig. 7. Histogram of the magnitudes of traction vectors from a single traction field. The fit to a log-normal distribution is shown by the line superimposed on the histogram. Inset: probability distribution of the logarithm of the traction magnitude. The crosses represent the data from a histogram with geometrically increasing bin width $(1,2,4$, and 8 times, etc.). The line is the fit to a normal distribution. In $A$ tractions from Fig. $6 A$ were used, whereas in $B$ the tractions were from Fig. $6 B$.

the weakest protrusions ( $t$-test, $P<0.05)$ at all times during the treatment.

We then asked whether traction in different protrusions starts to increase at the same time after stimulation with histamine. It was not always possible to determine accurately the difference in the traction onset in the protrusions of one cell because weak protrusions typically showed slow increase in traction and rather low signal-to-noise ratio. Nevertheless, it was
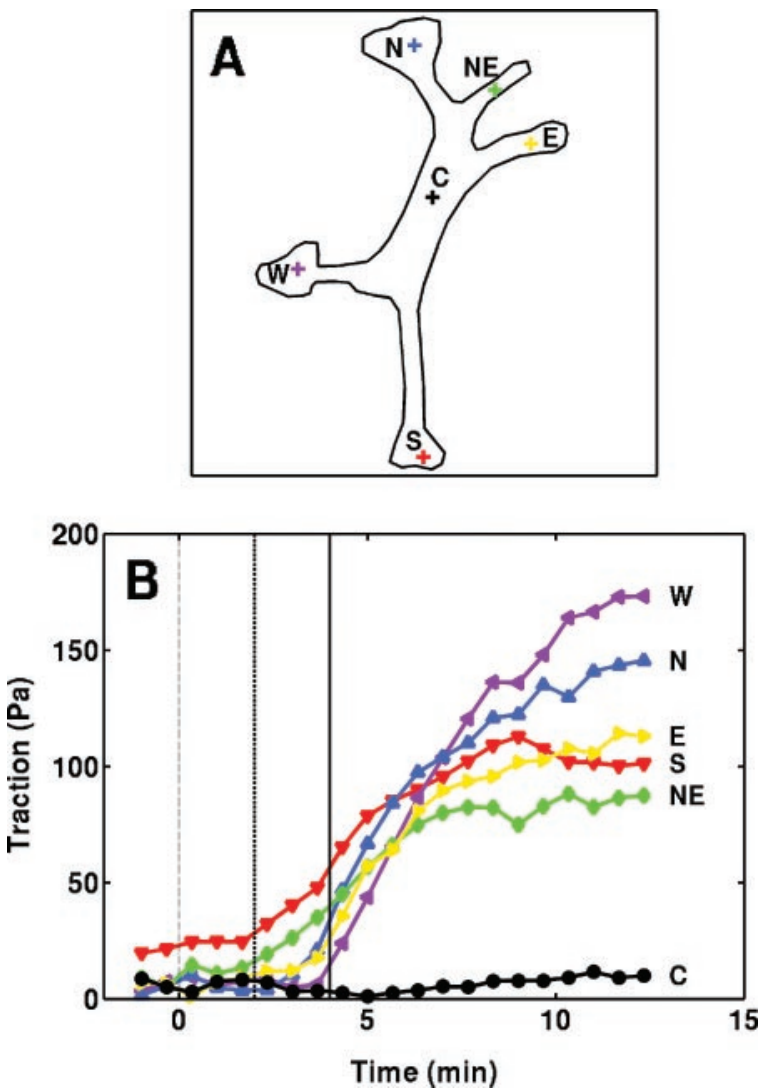

Fig. 8. Traction response to histamine varies across different small regions of a cell. A: outline of the cell from Fig. $2 A$. Six areas of $2.7 \times$ $2.7 \mu^{2}$ were chosen and marked as $N, N E, E, S, W$, and $C$. B: magnitude of traction exerted in the areas $N, N E, E, S, W$, and $C$ from $A$ as a function of time. Tractions were calculated using unconstrained FTTC. Vertical lines mark the application of histamine (0.1 $\mu \mathrm{M}$ at $t=0,1 \mu \mathrm{M}$ at $t=2 \mathrm{~min}$, and $10 \mu \mathrm{M}$ at $t=4 \mathrm{~min}$ ). possible to estimate the onset of traction increase in the strongest and the weakest protrusion in six cells. We estimated the half-time, i.e., the time when the traction in these protrusions was at its half-maximum value. The difference in the half-time values of the strongest and the weakest protrusion of the same cell was $1.3 \pm 0.8$ min (mean $\pm \mathrm{SD}, n=6$ ), ranging from 0.3 to $2.6 \mathrm{~min}$. Because the half-time of traction development was most reliably estimated for protrusions with large tractions, we estimated half-times for the strongest protrusion of each cell. The distribution of the half-time was broad: $5.2 \pm 1.8$ min (mean $\pm \mathrm{SD}$, $n=14$ ), range 2.3 to $9 \mathrm{~min}$. Taken together, these data imply that the onset of traction development varied between different cell protrusions.

\section{Scalar Representations of a Traction Field Show Changes in the Intensity and Polarity of Cell Contraction}

It is important to quantify the overall contractile intensity of the cell to compare different cells or different states of the same cell. In this work we used three ways to represent the complex traction map by a scalar: median traction, net contractile moment, and the strain energy. Figure $10 \mathrm{~A}$ shows the strain energy transferred from a histamine-treated cell to the elastic distortion of the gel, as a function of time. Similar curves were obtained for the median traction (increase from 60 to $150 \mathrm{~Pa}$ ), and the net contractile moment (increase from 7 to $18 \mathrm{pNm}$ ). All three measures of cell contraction decreased after isoproterenol treatment $(30-40 \%)$.

Figure $10 B$ shows a measure of the polarity of cell contraction, defined here as the ratio of the contractile moment along the principal axis of traction $\left(\mathrm{M}_{x x}\right)$ and the net contractile moment $\left(\mathrm{M}=\mathrm{M}_{x x}+\mathrm{M}_{y y}\right)$, as a function of time. Results of six representative histamine-treated cells are shown. The contraction along the principal axis of the cell constituted from $50 \%$ of the total contraction in symmetrical cells to $98 \%$ in highly elongated cells. The polarity either increased, decreased, or did not change after stimulation with histamine. The polarity decreased if the traction increased in the side protrusions of a cell and vice versa. The direction of the change in polarity thus depended 
Fig. 9. Mean traction differs markedly between different cell protrusions. Traction in cells treated with histamine $(A)$ and traction in cells treated with isoproterenol $(B)$. In each panel the time course of mean traction in the cell protrusion with the largest mean traction (Strong) and in the protrusion with the smallest mean traction (Weak) is shown. Protrusion regions were determined manually from the phase contrast images of cells. Vertical lines symbolize drug treatment ( $A$ : histamine, $0.1 \mu \mathrm{M}$ at $t=0,1 \mu \mathrm{M}$ at $t=2 \mathrm{~min}$, and $10 \mu \mathrm{M}$ at $t=4 \mathrm{~min} ; B$ : isoproterenol, $0.01 \mu \mathrm{M}$ at $t=0,0.1$ $\mu \mathrm{M}$ at $t=3 \mathrm{~min}, 1 \mu \mathrm{M}$ at $t=5.5 \mathrm{~min}$, and $10 \mu \mathrm{M}$ at $t=8.5 \mathrm{~min}$ ). Error bars represent SE. $A$ : $n=14$ cells, i.e., 14 strong protrusions and 14 weak protrusions; $B: n=5$ cells.
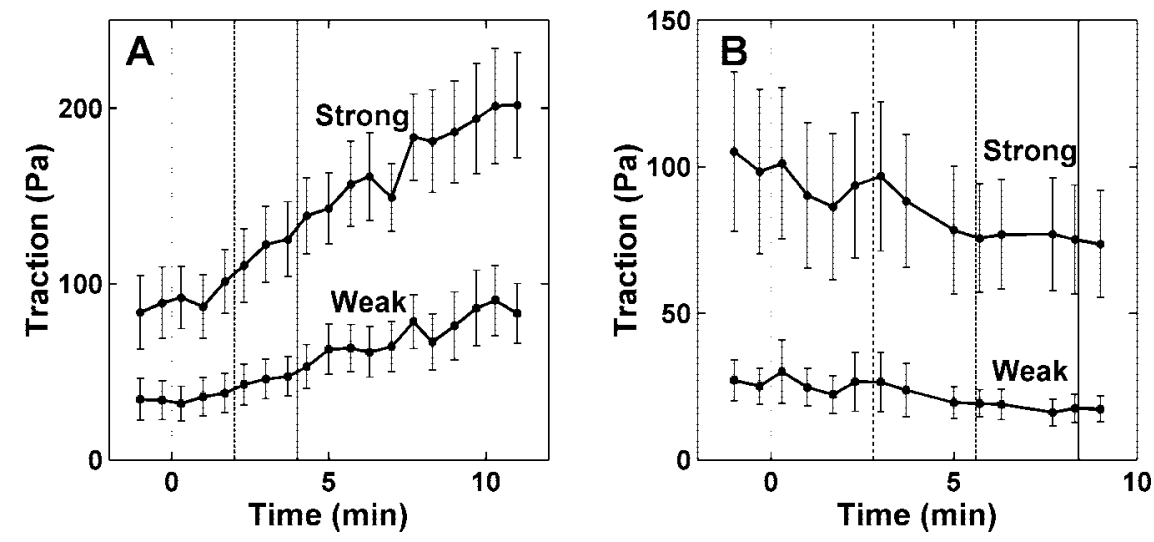

on the cell shape and differential traction responses in various cell protrusions.

\section{DISCUSSION}

The primary result of the current study is that tractions of adherent airway smooth muscle cells have distinct spatial and temporal distributions in response to contractile agonists; tractions at different regions of the cell increased to different magnitudes at different rates. In the following, we first discuss the techniques for quantifying the displacement fields, which are required for the determination of the traction fields. We then discuss possible underlying mechanisms of the spatial and temporal patterns of traction forces during contraction of HASM cells.

\section{ICM}

An important issue in the calculation of traction forces exerted by cells is how to determine the deformation of the flexible substrate in a reliable way. In this article we have developed and optimized the ICM for the determination of the displacement field. This method has substantial improvements compared with the method used in our previous work (3). The main new features of the ICM are summarized below.

In previous work (3) we used a simple semiautomated procedure for estimating the displacement field between two images of the fluorescent beads embedded in the gel. The idea was to locate small regions of a random pattern of beads after the pattern had been deformed. Images were divided into window areas of usually $64 \times 64$ pixels, and the cross-correlation was calculated between a pair of images. The location of the peak of the cross-correlation was used as the displacement vector of the window.

In this article the method for determining the displacement field was significantly refined. The underlying idea was the same, but a number of new features were incorporated. First, a hierarchical algorithm was introduced so that at the first level the global coarse displacement field is found. At each succeeding level the displacement field is refined locally. Therefore, as the spatial resolution of the calculated displacement field increases, the information obtained at a lower resolution is used in the search for the matching windows. Compared with the previously used method (3), this new algorithm increases both the speed of the calculations and the probability of finding the right matching window. It also allows for the use of several times smaller window areas and, hence, the detection of subtle local variations in the displacement field. Second, a few tests for the cross-correlation between a pair of windows were defined and optimized. The employment of these tests decreases the number of erroneous displacement vectors to a negligible fraction of the displacement field. Third, to get subpixel displace-
Fig. 10. A: strain energy in the gel conferred by cellular traction increases after stimulation by histamine. Mean strain energy is shown as a function of time. Error bars represent SE; $n=14$ cells. $B$ : cells show either increase, decrease, or no change in the polarity of contraction after stimulation with histamine. Ratio of the contractile moment along the principal axis of traction $\left(\mathrm{M}_{x x}\right)$ and the net contractile moment $\left(\mathrm{M}=\mathrm{M}_{x x}+\mathrm{M}_{y y}\right.$ ) is shown as a function of time. Results of 6 representative cells are shown. Vertical lines symbolize histamine treatment $(0.1 \mu \mathrm{M}$ at $t=$ $0,1 \mu \mathrm{M}$ at $t=2 \mathrm{~min}$, and $10 \mu \mathrm{M}$ at $t=4$ $\min )$.
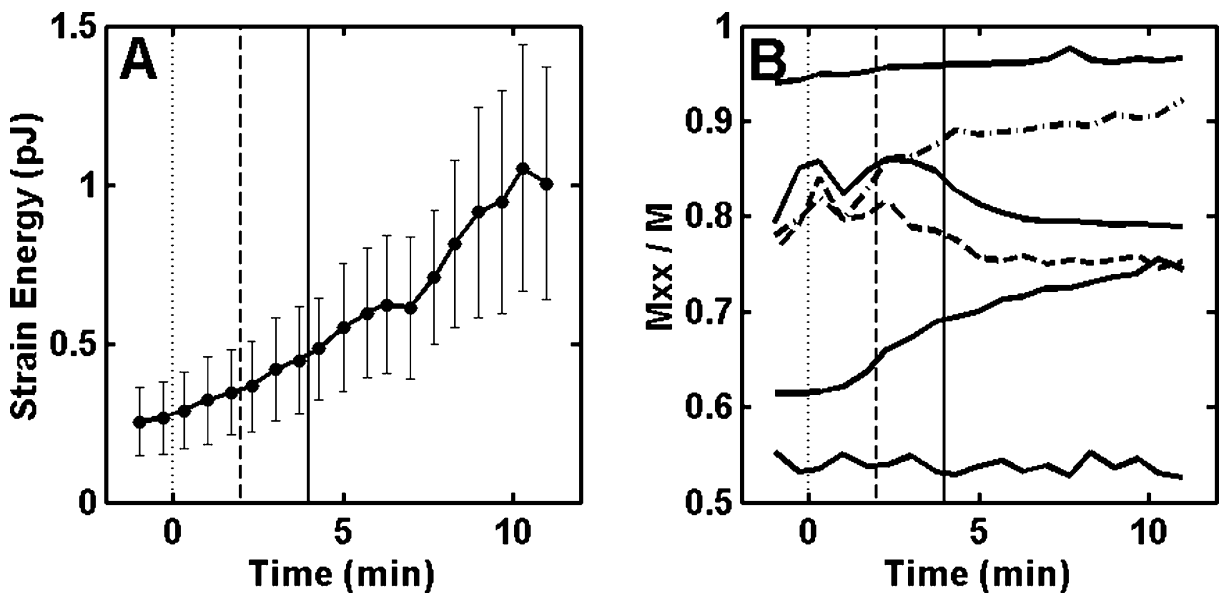
ment values, the values of the correlation function in the immediate neighborhood of the pixel with the highest value were fit to a second-order polynomial, and the maximum of the polynomial was used as the displacement value. The ability to detect displacements smaller than a pixel implies that the traction fields can be determined even when the deformation of the substrate under the cell is small, as in the case of stiff gels or weakly contracting cells. Finally, the noise was reduced by passing the displacement field through a low-pass filter. This is an important issue because the noise from the displacement field gets amplified when calculating the traction field. The filtering used in the ICM is a clear and simple way to smooth the data at the level of the displacement field so that the calculation of the traction field from the final displacement field remains exact. Taken together, the main advantages of the ICM compared with the previously used method (3) are higher accuracy, higher spatial resolution, detection of displacements of subpixel magnitude, and shorter time needed for the calculations.

The way of presenting data is another noteworthy topic. With the aim of showing as much information as possible and making the presentation clear at the same time, we have refined the visualization of the results. The displacement field is presented in such a way that the resulting image contains the information about the absolute magnitudes of the displacement vectors together with their direction and the relative magnitude. Moreover, the map between the colors and the displacement values is chosen to be such that the isodisplacement curves are easily visualized. The representation of traction maps is analogous.

Methods similar to the ICM for determining the deformation of a pattern based on correlation have been used in a wide variety of fields, from material science to medicine (19). The underlying idea is always the same, but the specific implementation depends on the features of the images used, the patterns that are to be matched, and the type and length scale of the expected deformation. Therefore, a new method has to be developed and optimized specifically for every new problem. The ICM yielded reasonable displacement fields in our experiments. However, certain experimental conditions, such as images with too few beads or clustered beads, as well as a change of focal plane during the experiment, may introduce errors in the displacement field.

An overall goodness of an estimated displacement field can be obtained by looking carefully at the images; if one looks at the images in a fast sequence, one notices movement of some groups of beads. The displacement field calculated by the ICM should show the same movement. We tested the ICM visually. The visually estimated displacement field was almost always in agreement with the displacement field calculated by the ICM. If this was not the case, free parameters in the ICM were changed appropriately until the calculated displacement field approached the visually determined one.
To test the ICM more rigorously, we performed Monte Carlo simulations. The simulations showed that the errors in the displacement fields are reasonably small for images with large enough number of beads, for small enough window size, and for the underlying displacement function that does not change drastically over a window.

The ICM can be used with any kind of deformable substrate that has a random pattern on it. The random pattern can be naturally occurring, inserted in the material, or applied to the surface of the material (29). A large selection of suitable random patterns makes it possible to design materials other than the polyacrylamide gel with imbedded beads for the determination of cellular traction forces.

After determining the displacement field by the ICM, we used FTTC for calculating the traction fields exerted by cells (3). Cellular traction forces were assumed to be in the plane of the interface between the cell and the substrate. Indeed, the cells investigated here were well spread on the surface of the gel. The average height-to-length ratio of the cell was $\sim 1 / 50$. These cells exert traction forces along the axis of their contractile filaments; hence, the force component perpendicular to the gel surface is negligible compared with the in-plane components.

Note here that there is a potential limitation of our approach, because most contractile cells are embedded in a three-dimensional (3D) matrix in vivo and subject to a complex 3D pattern of forces, whereas the approach used here investigates cells cultured on the two-dimensional (2D) surface of a gel. This might limit the relevance of the conclusions drawn from these experiments to the cells in vivo. It is possible in principle to generalize this $2 \mathrm{D}$ approach to $3 \mathrm{D}$, but there are serious technical limitations in multiple image acquisitions, extension of correlation maps to obtain 3D displacement fields, and new mathematics for the inversion of the $3 \mathrm{D}$ problem to recover the tractions at the nonplanar cell boundary with the substrate.

Furthermore, cultured cells are devoid of gap junctions, autocrine and paracrine factors that affect them in vivo. These cells undergo dedifferentiation and reorganization of the contractile apparatus. Therefore, one should be cautious when inferring the properties of cells in vivo from these experiments.

Despite the potential limitations of the approach, this work shows that FTTC together with the ICM is a powerful and easily applicable tool for estimating the cellular traction forces with high spatial and temporal resolution. Moreover, it is also a useful method for studying the effect of drugs and chemicals on the contraction and relaxation patterns of different types of cells. The spatial resolution of our method, i.e., the distance between neighboring traction vectors, was chosen to be $2.7 \mu \mathrm{m}$. However, higher resolution can be achieved with a higher number of fluorescent microbeads per image area. The spatial resolution of the method is ultimately limited by the resolution of light microscopy. Combining FTTC with methods for visualizing relevant mediators of cell contraction is likely to 
provide a better understanding of the mechanisms by which cells develop mechanical forces. Analysis of the spatial distribution of cellular signaling events (e.g., bound receptors, open channels, cytoplasmatic calcium) and contractile elements (distribution of cytoskeletal filaments and focal adhesion complexes) may be paired directly with the spatial analysis of traction. For example, a superposition of the traction map and a map of contractile filaments might lead to identification of the principal contractile elements in the cell. Likewise, the effect of the number and kind of attachments on the traction pattern could be explored by varying the coating density of the matrix and by using various species of matrix molecules (e.g., fibronectin, laminin, and vitronectin).

The temporal resolution of our method is not limited to $40 \mathrm{~s}$, as used in this work. It is limited only by the image acquisition speed of the camera $(50 \mathrm{~ms})$. Thus the method presented here may be used to study the effects of the dynamics of attachment proteins, signaling proteins, and motor proteins on the generation and distribution of traction forces.

\section{Traction Maps of Single Smooth Muscle Cells}

We have presented examples of traction fields generated by HASM cells before and after stimulation with either histamine or isoproterenol. All the traction fields show similar features. The tractions are largest in the far ends of the cellular extensions, possibly because those might be the sites of strong focal adhesions and ends of stress fibers. The tractions are directed towards the nucleus, where their values are small. The lack of traction under the nucleus may be interpreted as follows: the majority of the focal adhesion complexes are typically found at the ends of the cell protrusions, whereas only a small fraction of focal adhesions are found under the nucleus [see Fig. 9 in (31) and note that actin filaments terminate in cell protrusions and not under the nucleus]. The lack of attachments under the nucleus implies the lack of force transmitted from the cell to the substrate in that region. However, negligible traction near the middle of the cell may also be associated simply with continuity of the traction field that is uniformly pulling inwards, which implies zero or small tractions somewhere in the interior, independent of the density of focal adhesions or cell-substrate interaction.

The magnitudes of traction vectors of a single traction field display a log-normal distribution. A log-normal distribution arises when the causes of variability have multiplicative effects, as opposed to additive effects which lead to a normal distribution (20). The finding that the tractions follow a log-normal distribution implies that the average traction is not a robust maximum likelihood estimator of tractions. Instead, the median traction should be used to characterize the typical interfacial force between the cell and the substrate.

Besides the median traction, complex traction maps can be reduced to several other scalar numbers that characterize the contractile state of the cell, e.g., the strain energy in the gel conferred by cellular tractions and the net contractile moment of the cell. Such measures allow for a comparison of the strength of the cell contraction before and after mechanical and biochemical manipulations, as well as the contraction of different cells. Strain energy is a particularly useful measure of the contractile strength. For instance, a single actin-myosin interaction can generate a maximum energy of about $10^{-8} \mathrm{pJ}(9,21)$. The strain energy we measured in a single cell stimulated with $10 \mu \mathrm{M}$ histamine is of the order of $1 \mathrm{pJ}$. Thus there must be about 100 million actin-myosin cross bridges working together to generate a comparable amount of strain energy. This suggests that some of the forces are transmitted from the depth of the cell to the focal adhesion sites.

We found spatial and temporal heterogeneity in traction development after treatment with histamine. Heterogeneity in traction response was also observed during treatment with the bronchodilator isoproterenol, where it was less prominent because of the weak effect of isoproterenol treatment ( $\sim 30 \%$ change in traction) compared with the histamine treatment $(\sim 150 \%$ change in traction). During treatment with histamine, traction forces start to increase at different times in different cell protrusions. Furthermore, traction reaches markedly different magnitudes in different regions of a cell. What are the possible underlying mechanisms of the spatial and temporal differences in the generation of traction in a cell? There are several possibilities. First, different parts of a cell could react to histamine differently because of an inhomogeneous distribution of histamine in the medium. This, however, is unlikely. The concentration of histamine equilibrates fast through the mixing of the injected histamine solution with the medium. Furthermore, opposite parts of a cell reacted simultaneously, which is not consistent with a gradient of histamine concentration in the medium. The second possibility is that histamine receptors and the associated membrane components, e.g., G proteins, phospholipase C, phosphatidyl inositol 4,5-bisphosphate (13) could be unevenly distributed in the cell membrane. Although we do not have evidence to support this hypothesis, we cannot rule it out.

The third possibility could be related to the regional differences in distribution and activation of the contractile apparatus and focal adhesions. It has recently been shown in nonmigrating fibroblasts that the magnitudes and directions of local contact forces are correlated with the size and orientation of the focal adhesions (1). It is possible that the temporal sequence of increase in traction after stimulation with histamine in HASM cells reflects the temporal activation of the contractile components in focal adhesion complexes in these regions and, subsequently, the growth of the focal adhesions. However, in migrating cells, it could be very different. Beningo et al. (2) showed that strong tractions are generated by nascent focal adhesions in migrating fibroblasts and not by large focal adhesions. 
Focal adhesion proteins have been suggested as key mediators of actin remodeling in response to agonists (8). Measurements of the actin dynamics in cultured smooth muscle cells (30) showed that actin kinetic parameters display a cell-wide gradient. The unbinding rate of actin near the plasma membrane at the cell-substrate contact regions, as measured using total internal reflection microscopy and fluorescence recovery after bleaching, was found to be spatially nonuniform throughout the cell (30). In some cell regions actin kinetics was fast, whereas in other regions it was slower. The difference in actin kinetics might be related to dynamics of focal adhesion complexes and reflect regional differences in focal adhesion activity. Actin polymerization may in turn contribute to force development and maintenance through attachments of actomyosin filaments to focal adhesion complexes. Regional differences in the kinetics of actin and focal adhesion complexes might generate regional differences in cellular traction fields.

Smooth muscle contraction is primarily regulated by calcium ions through the activation of myosin light chain kinase (MLCK; reviewed in ref. 10). Calcium concentration is spatially inhomogeneous throughout the cytoplasm: local $\mathrm{Ca}^{2+}$ transients, named $\mathrm{Ca}^{2+}$ sparks, occur spontaneously in smooth muscle cells (14, 15, 25). Pabelick et al. (24) showed that spark amplitude and occurrence varies considerably across different regions of the cells. Exposure to agonists triggers $\mathrm{Ca}^{2+}$ oscillations that always originate from the sites with highest spark incidence (24). The spatially inhomogeneous $\mathrm{Ca}^{2+}$ concentration might induce a spatially distinct activation of MLCK and, hence, spatial differences in myosin activity and the traction exerted on the cell substrate.

Smooth muscle contraction is also modulated by factors other than $\mathrm{Ca}^{2+}$. One of these factors is the small $\mathrm{G}$ protein Rho, which positively regulates the level of myosin phosphorylation (16) and activates actin polymerization and stress fiber assembly $(12,32)$. Spatial inhomogeneity in Rho activity in smooth muscle cells was found by Miyazaki et al. (22). They reported that Rho-dependent pathway induces myosin phosphorylation in cell cortical regions, whereas MLCK phosphorylates myosin in the inner space of the cell. A few minutes after stimulation the phosphorylation was sustained in cortical regions but not in central fibers (22). Spatial differences in Rho activity could influence traction force patterns. For example, it is possible that in flat cell regions actomyosin filaments interact with the mediators of Rho-pathway located in the plasma membrane and, hence, exert sustained force.

Do regional differences in traction reflect important functions of smooth muscle cells in tissue? Smooth muscle cells manifest mechanical plasticity as the result of remodeling of the contractile apparatus in response to changes in cell shape (11). Contractility of these cells adapts to changes in cell length and load, which is crucial for their function in vivo in the regulation of the diameter of hollow organs. Regional differences in traction response in cultured smooth muscle cells might be an aspect of the constant reorganization and plasticity of these cells.

\section{Conclusions}

In summary, we have measured highly detailed traction fields of HASM cells. We have determined the development of traction during stimulation with histamine and the relaxation of traction during treatment with isoproterenol. The cells showed regional differences in the development of traction. The onset of traction increase and the maximum traction induced by histamine varied markedly between different cell protrusions, whereas the nuclear region response was the smallest. Our results suggest that intracellular contractile units, contractile regulators, and focal adhesion complexes process contractile stimuli at different rates in different regions of the cell. Localized stimulation of specific mediators of traction, combined with ICM and FTTC, may help to elucidate how the different players of the contractile machinery work together.

We thank J.J. Fredberg, B. Fabry, and E.J. Millet for productive discussions. We thank S.M. Mijailovich for help in the early course of this work. Cells were kindly supplied by R. Panettieri.

This work was supported by The Ministry of Science and Technology of the Republic of Croatia, National Aeronautics and Space Administration grant NAG2-1509, and National Heart, Lung, and Blood Institute grant HL-33009.

\section{REFERENCES}

1. Balaban NQ, Schwarz US, Riveline D, Goichberg P, Tzur G, Sabanay I, Mahalu D, Safran S, Bershadsky A, Addadi L, and Geiger B. Force and focal adhesion assembly: a close relationship studied using elastic micropatterned substrates. Nat Cell Biol 3: 466-472, 2001.

2. Beningo KA, Dembo M, Kaverina I, Small JV, and Wang YL. Nascent focal adhesions are responsible for the generation of strong propulsive forces in migrating fibroblasts. J Cell Biol 153: 881-888, 2001.

3. Butler JP, Tolić-Nørrelykke IM, Fabry B, and Fredberg JJ. Traction fields, moments, and strain energy that cells exert on their surroundings. Am J Physiol Cell Physiol 282: C595C605, 2002.

4. Chen CS, Mrksich M, Huang S, Whitesides GM, and Ingber DE. Geometric control of cell life and death. Science 276: 14251428, 1997.

5. Chen J, Fabry B, Schiffrin EL, and Wang N. Twisting integrin receptors increases endothelin-1 gene expression in endothelial cells. Am J Physiol Cell Physiol 280: C1475-C1484, 2001.

6. Chicurel ME, Singer RH, Meyer CJ, and Ingber DE. Integrin binding and mechanical tension induce movement of mRNA and ribosomes to focal adhesions. Nature 392: 730-733, 1998.

7. Dembo M and Wang YL. Stresses at the cell-to-substrate interface during locomotion of fibroblasts. Biophys J 76: 23072316, 1999.

8. Gerthoffer WT and Gunst SJ. Focal adhesion and small heat shock proteins in the regulation of actin remodeling and contractility in smooth muscle. J Appl Physiol 91: 963-972, 2001.

9. Guilford WH, Dupuis DE, Kennedy G, Wu J, Patlak JB, and Warshaw DM. Smooth muscle and skeletal muscle myosins produce similar unitary forces and displacements in the laser trap. Biophys J 72: 1006-1021, 1997.

10. Gunst SJ and Tang DD. The contractile apparatus and mechanical properties of airway smooth muscle. Eur Respir $J 15$ : 600-616, 2000. 
11. Halayko AJ and Solway J. Molecular mechanisms of phenotypic plasticity in smooth muscle cells. J Appl Physiol 90: 358368, 2001.

12. Hall A. Rho GTPases and the actin cytoskeleton. Science 279: 509-514, 1998.

13. Hall IP. Second messengers, ion channels and pharmacology of airway smooth muscle. Eur Respir J 15: 1120-1127, 2000.

14. Jaggar JH, Porter VA, Lederer WJ, and Nelson MT. Calcium sparks in smooth muscle. Am J Physiol Cell Physiol 278: C235-C256, 2000.

15. Janiak R, Wilson SM, Montague S, and Hume JR. Heterogeneity of calcium stores and elementary release events in canine pulmonary arterial smooth muscle cells. Am J Physiol Cell Physiol 280: C22-C33, 2001.

16. Kimura K, Ito M, Amano M, Chihara K, Fukata Y, Nakafuku M, Yamamori B, Feng J, Nakano T, Okawa K, Iwamatsu A, and Kaibuchi K. Regulation of myosin phosphatase by Rho and Rho-associated kinase (Rho-kinase). Science 273: 245-248, 1996.

17. Landau LD and Lifshitz EM. Theory of Elasticity (3rd ed.) Oxford, UK: Pergamon, 1986.

18. Li Y, Hu ZB, and Li CF. New method for measuring Poisson ratio in polymer gels. J Appl Polymer Sci 50: 1107-1111, 1993.

19. Likar B and Pernuš F. Registration of serial transverse sections of muscle fibers. Cytometry 37: 93-106, 1999.

20. Limpert E, Stahel WA, and Abbt M. Log-normal distributions across the sciences: keys and clues. Bioscience 51: 341-352, 2001.

21. Linari M, Dobbie I, Reconditi M, Koubassova N, Irving M, Piazzesi G, and Lombardi V. The stiffness of skeletal muscle in isometric contraction and rigor: the fraction of myosin heads bound to actin. Biophys J 74: 2459-2473, 1998.

22. Miyazaki K, Yano T, Schmidt DJ, Tokui T, Shibata M, Lifshitz LM, Kimura S, Tuft RA, and Ikebe M. Rho-dependent agonist-induced spatio-temporal change in myosin phos- phorylation in smooth muscle cells. J Biol Chem 277: 725-734, 2002.

23. Munevar S, Wang YL, and Dembo M. Traction force microscopy of migrating normal and H-ras transformed 3T3 fibroblasts. Biophys J 80: 1744-1757, 2001.

24. Pabelick CM, Prakash YS, Kannan MS, and Sieck GC. Spatial and temporal aspects of calcium sparks in porcine tracheal smooth muscle cells. Am J Physiol Lung Cell Mol Physiol 277: L1018-L1025, 1999.

25. Pabelick CM, Sieck GC, and Prakash YS. Significance of spatial and temporal heterogeneity of calcium transients in smooth muscle. J Appl Physiol 91: 488-496, 2001.

26. Panettieri RA, Murray RK, DePalo LR, Yadvish RA, and Kotlikoff MI. A human airway smooth muscle cell line that retains physiological responsiveness. Am J Physiol Cell Physiol 256: C329-C335, 1989.

27. Pelham RJ and Wang YL. Cell locomotion and focal adhesion are regulated by substrate flexibility. Proc Natl Acad Sci USA 94: 13661-13665, 1997.

28. Singh A. Optic Flow Computation: A Unified Perspective. Los Alamitos: IEEE Computer Society Press, 1991.

29. Sun Z, Lyons JS, and McNeill SR. Measuring microscopic deformations with digital image correlation. Optics Lasers Engr 27: 409-427, 1997.

30. Sund SE and Axelrod D. Actin dynamics at the living cell submembrane imaged by Total Internal Reflection Fluorescence Photobleaching. Biophys J 79: 1655-1669, 2000.

31. Wang N, Tolić-Nørrelykke IM, Chen J, Mijailovich SM, Butler JP, Fredberg JJ, and Stamenovic D. Cell prestress. I. Stiffness and prestress are closely associated in adherent contractile cells. Am J Physiol Cell Physiol 282: C606-C616, 2002.

32. Watanabe $\mathbf{N}$, Kato T, Fujita A, Ishizaki T, and Narumiya S. Cooperation between mDia1 and ROCK in Rho-induced actin reorganization. Nat Cell Biol 1: 136-143, 1999.

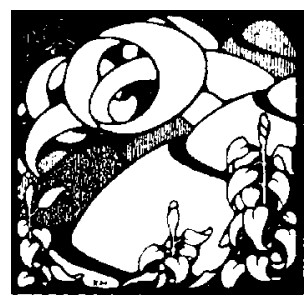

